\title{
Application of Mathematical Modelling on the Spread of Chicken Pox Disease

\author{
(A Case Study of Nayinawa Clinic Damaturu, Yobe State)
} \\ Umar Yusuf Madaki ${ }^{1 *}$, Habiba Adamu Manu ${ }^{1}$, Alhaji Abdullahi Gwani ${ }^{2}$, Edeghagba Eghosa Elijah ${ }^{2}$
}

${ }^{1}$ Department of Mathematics and Statistics, Yobe State University, Nigeria

${ }^{2}$ Department of Mathematical Sciences, Bauchi State University, Gadau, Nigeria

DOI: $10.36347 /$ sipms.2020.v07i10.004

| Received: 21.01.2020 | Accepted: 28.01.2020 | Published: 11.10.2020

*Corresponding author: Umar Yusuf Madaki

Abstract

Original Research Article

Chickenpox was declared an emergency in Nigeria and strategies for the control of chickenpox in Nigeria were adopted to reduce its prevalence to a level at which the disease will no longer constitute public health problems. In this work, we presented a deterministic (SEIR susceptible, expose/latently infected, infectious and recovered) model incorporating the method of control adopted by national chickenpox and leprosy control program. We established the disease free and the endemic equilibrium states and carried out the stability analysis of the disease. Free and the equilibrium state of the model to have an insight into the dynamics of the model. We found out that the disease free equilibrium state is stable. The numerical results showed that it would be very difficult to completely eradicate chickenpox from Damaturu-Yobe and the country Nigeria at large.

Keywords: Chickenpox, Differential Equation, Mathematical Modeling, Susceptible, Infectious, Infected, Recovered. Copyright @ 2020: This is an open-access article distributed under the terms of the Creative Commons Attribution license which permits unrestricted use, distribution, and reproduction in any medium for non-commercial use (NonCommercial, or CC-BY-NC) provided the original author and source are credited

\section{INTRODUCTION}

Chickenpox is common and mostly affects children, although you can get it at any age. It usually gets better by itself within a week. No doubt, the history of humankind has been shaped by the pitiless outbreaks of infectious disease pandemics, whole nations and civilizations have been copied off the map through the ages. Chickenpox (chicken pox), also known as varicella, is a highly contagious infection caused by the varicella zoster virus. Although uncomfortable, most people recover within 1-2 weeks. There is a blister-like rash, which first appears on the face and trunk, and then spreads throughout the body. Although not life threatening, complications can arise.

Chickenpox, also known as varicella, is a highly contagious disease caused by the initial infection with varicella zoster virus (VZV). The disease results in a characteristic skin rash that forms small, itchy blisters, which eventually scab over. It usually starts on the chest, back, and face. It then spreads to the rest of the body.

Chickenpox is an airborne disease, which spreads easily through the coughs and sneezes of an infected person. It may be spread from one to two days before the rash appears until all lesions have crusted over. It may also spread through contact with the blisters. Those with shingles may spread chickenpox to those who are not immune through contact with the blisters.

Chickenpox occurs in all parts of the world. In 2013, there were 140 million cases of chickenpox and herpes zoster worldwide. Before routine immunization, the number of cases occurring each year was similar to the number of people born. Since immunization, the number of infections in the United States has decreased nearly $90 \%$. In 2015 , chickenpox resulted in 6,400 deaths globally down from 8,900 in 1990. Death occurs in about 1 per 60,000 cases. Chickenpox was not separated from smallpox until the late 19th century. In 1888, its connection to shingles was determined. The first documented use of the term chicken pox was in 1658. Various explanations have been suggested for the use of "chicken" in the name, one being the relative mildness of the disease. 


\section{BACKGROUND OF THE STUDY}

In Athens from 425 to $430 \mathrm{BC}$ set of the peridean golden era, the "COCOLIZTLI" epidemics which occurred during the $16^{\text {th }}$ Century resulted in 13 million death decimating Mesoamerican native population, the black bubonic plague burst in Europe in 1348 and is estimated to have killed over 25 million people just in five years. The pandemic influenza Virus of 1918 - 1919 swept through America, Europe, Asia and Africa Smashing the globe. The death toll was around 40 million people. Two - and year less fever pandemic followed in the next decade. The 1975 and 1963 influenza pandemic resulted in two and one million death respectively B. AR.

In the last decade emergency and re-emerging pandemic such as Aids, Chickenpox and Malaria cause death of millions of people each year. According to the UNAIDS report on the global Aids epidermis an estimated 34 million people including 3.4 million children were living with the HIV worldwide at the end of 2010 while the related death and the near infections were 1.8 and 2.7 million respectively, problems stems mainly from two reasons.

- The contentious everlasting mutation of viruses

- The complexity in the disease transmission

Mechanism unfortunately the odds are that in a real crises even if the researcher's succeeded to come up with a vaccine tailor-made for an emerged virus strain it is doubtful that it would step a pandemics. Chickenpox is very itchy and can make children feel miserable, even if they do not have many spots. Chickenpox is usually much worse in adults. It is possible to get chickenpox more than once, although it is unusual.

The complex multi-scale interplay between the lost factor ranging from the micro-host pathogen and individual scale host, host or macro-scale ecological, social, economic and demographic condition across the globe complicated, by technical issued such time long between vaccine prototype development and commercial distribution and production imposes a real impediment to our central strategy partial mathematical and statistical model are playing a most valuable role shading light on the problem and for helping make decision.

\section{STATEMENT OF THE PROBLEM}

The emergence and re-emergence of pandemic such as Chickenpox that causes millions of people especially children each year is the point of concern. Chickenpox, a characteristic vesicular rash, is a respiratory infection caused by the varicella-zoster virus, which can be transmitted through airborne droplets and through the contact with the patient's fresh blisters or mucosal secretions.
Meanwhile, it is a common acute and highly contagious disease during childhood, and $90 \%$ of susceptible children will be infected and most of which will not be seeking medical attention. However, serious complications mainly including neurological involvement and secondary bacterial infections and even severe death were observed both among cases of children and adults. The risk for disease increases with age but is also elevated in infants and the hypoimmunity. The present study focuses on the analysis of the vaccine efficacy and does not effectively prevent the occurrence and outbreak of chickenpox. Therefore, carrying out an early warning of chickenpox plays an important role in taking targeted measures in advance as well as preventing the outbreak.

Studies have indicated that the logistic differential equation model can well demonstrate the epidemic characteristics of epidemic outbreaks, give the point where the early epidemic rate change from slow to fast, and is suitable for the simulation of epidemic features in epidemic cycles of infectious diseases. What is more, the study has illustrated that chickenpox has significant seasonality and periodicity; thus, it is suitable for preseasonal warning research.

Therefore, our study intends to use the logistic differential equation model to fit the weekly data of chickenpox in Damaturu from August 2018 to 2019, to discuss the application of the model, calculate the inflection point of epidemic speed changes in each period of chickenpox and get the warning week finally, and thus, provide a scientific basis for early warning of chickenpox. Here we are aim to systematically the theoretical studies undertaken so far to study the impact of individual on the epidemiology of infection diseases. Firstly, we propose a classification system that we believe is useful to help bring order to complex diversity of models.

Secondly, we aim to assess the contribution that the model make towards understand dynamic control of disease, as well as suggestion that follows as to how response to outbreak could be measured and quantify in a reasonable way. All the effort made by the previous scientist such as

Helbing et al., [1] and Wilson [2], Boots and Ferguson [3], Hatchett et al., [4] were able to control the spread of contagious disease such as chickenpox based on public health measure. This study 2019 is centrally on determining a predicting the rate at which a contagious disease spread with time in a given community of host, which was not, made clearing by their work. 


\section{RESEARCH QUESTION}

For the fact that differential equation depict the relationship between parameters and their changes with respect time hence the need to answer these questions.

- Is it possible that there is a big outbreak infecting a positive fraction of the host community?

- How many will be infected if the epidemic takes off?

- What is the epidemic level?

- What is the effect of vaccinating a given community of host prior to the arrival of diseases?

\section{RESEARCH OBJECTIVE}

To determine the rate at which a contagious disease (chickenpox) spread with time and to be able to control it affects over a short period within the case study.

\section{SIGNIFICANCE OF THE STUDY}

The emergence and re-emergence of chickenpox outbreak in the temperate state of this country that causes death to millions of children especially those below five (5) years of age is main point of concerned. Hence, the need for this research.

\section{RESEARCH HYPOTHESIS}

A linear differential equation using variable separable and logistic models to predicts the exponential growth rate of chickenpox in a community of host if no isolation was made especially in such closed population of Nayinawa community, DamaturuYobe, Nigeria.

\section{SOME REVIEWS}

Amerasinghe and Penelope V [5], Generalized seasonal autoregressive integrated moving average models for count data literature with application to malaria time series with low case numbers. Chickenpox, a characteristic vesicular rash, is a respiratory infection caused by the varicella-zoster virus, which can be transmitted through airborne droplets and through the contact with the patient's fresh blisters or mucosal secretions. Meanwhile, it is a common acute and highly contagious disease during childhood, and $90 \%$ of susceptible children will be infected and most of which will not be seeking medical attention.

Wang YW et al., [6], However, serious complications mainly including neurological involvement and secondary bacterial infections and even severe death were observed both among cases of children and adults. The risk for disease increases with age but is also elevated in infants and the hypo immunity. The present study focuses on the analysis of the vaccine efficacy and does not effectively prevent the occurrence and outbreak of chickenpox. Therefore, carrying out an early warning of chickenpox plays an important role in taking targeted measures in advance as well as preventing the outbreak.

Najjar [7], studies have indicated that the logistic differential equation model can well demonstrate the epidemic characteristics of epidemic outbreaks, give the point where the early epidemic rate change from slow to fast, and is suitable for the simulation of epidemic features in epidemic cycles of infectious diseases. What is more, the study has illustrated that chickenpox has significant seasonality and periodicity; thus, it is suitable for pre-seasonal warning research.

Therefore, our study intends to use the logistic differential equation model to fit the weekly data of chickenpox in Damaturu from 2008 to 2018, to discuss the application of the model, calculate the inflection point of epidemic speed changes in each period of chickenpox and get the warning week finally, and thus, provide a scientific basis for early warning of chickenpox $\mathrm{Z}$.

Ogunjimi et al., [8], reported a new individualbased model to assess the effect of childhood varicella vaccination on shingles cases that factors in the immune responses to varicella infection. The model suggests that re-exposure to the varicella virus through contact with infected people would only provide extra protection for about two years; this is much shorter than previous predictions that suggested it might last 20 years. The model also predicts that implementing a varicella vaccination program for children would almost double the number of shingles cases 31 years later. But this increase would be temporary.

According to Koriko and Yusuf [9], in their model "mathematical model to simulate chickenpox disease population dynamics" chickenpox disease population dynamics was presented, their model was compartmentalized as appropriate and the resulting model equation were solved numerically with different instance of disease transmission were presented and addition, the graphical profile of the various sub population with time were presented and equilibrium of the system were established and analyzed for stability .

Inyama [10], consider the role of vaccination of newborn babies against chickenpox and treatment of both latently and actively infected individual in controlling the spread of chickenpox, which was mathematically model, based on the standard SEIR model. The disease-free equilibrium state of the model was established and its stability of the disease -free the Routh-Hurwitz theorem. The result of their analysis of the stability of the disease - free equilibrium state shows that chickenpox can totally be eradicate if effort is made to ensure that the sum of the rate of recovery of 
the latent class, the rate at which latently effected Individuals becomes actively infected and the rate of natural death, must have a lower bound.

Engel [11], also commented that Holmes approach was causal may be bidirectional or even cyclic, thus their works fail to produce statistical convincing studies. On this point the researcher, agree with Engels view regarding the gap in Holmes work.

Tracy et al., [12], present a Meta - analysis of the literature on stress and immunity in humans. The way neuroendocrine mechanism sand health practice might alternate stress has been discussed, evidence for the relations between stress and both functional and enumerative immune measures have been presented. The authors further stated that, stressor duration is important for immune outcomes, and interpersonal events are related to alterations incident immune parameters than social events. However, their work lacked a qualitative and numerical analysis, which this study has covered.

Van Damme et al., [13], Using a longitudinal mixture modeling approach, they found out that $25 \%$ and $17 \%$ of re-exposed grandparents showed a boosting of VZV-specific CD4+ IL-2-producing T-cells and VZV-specific antibodies, respectively. The antibody boosting occurred exclusively in cytomegalovirus (CMV) IgG-positive participants are largely driven by the more intensive intergenerational contacts between children, their parents, grandparents, and an assumed direct inverse proportionality between the number of contacts with chickenpox cases and the probability of developing herpes zoster.

Caroline et al., [14], used two mathematical models to explore the role of the contact structure of the population, and found out that in declining epidemics, localized outbreaks may occurs as a result of contact heterogeneity even in the absence of host or strain variability. They discussed the implications of their finding for chickenpox control in low incidence setting. Thus, some local outbreaks can be expected to occur even in the absence of these sources of individual heterogeneity, because of the contact structure of the population. Furthermore, their findings suggest that non - random mixing has a substantial effect on clustering of the disease in low incidence areas, hence concluding that's non - random mixing in a population leads to the emergency of local disease clusters in declining chickenpox epidemics which occurs even when the initial condition are spatially homogeneous and individual heterogeneity is excluded. Their work is relevant to this study in understanding the contact structure of chickenpox dynamics although this study specially models the effect of stress on the transmission dynamics of chickenpox.
Cohen and Judith Breuer [15], Chickenpox is extremely contagious, more than $90 \%$ of unvaccinated people will become infected during their lifetime, but infection occurs at different ages in different parts of the world. In the US, the UK, and Japan, more than $80 \%$ of people have been infected by the age of 10 years and by the age of 20 to 30 years in India, South East Asia, and the West Indies. It is usually a mild and self-limiting disease, but it can be severely complicated by pneumonitis or disseminated disease in some individuals, particularly neonates and those who are immune-compromised.

Peer J et al., [16], developed an agent-based model representing VZV disease, transmission, vaccination states and coverage, waning and boosting of immunity in a stylized geographic area, utilizing a distance-based network. We derived parameters from literature, including modeling, epidemiological, and immunology studies. We calibrated our model to the age-specific incidence of shingles and chickenpox prior to vaccination to derive optimal combinations of duration of boosting (DoB) and waning of immunity.

\section{SIGN AND SYMPTOM}

The early (prodromal) symptoms in adolescents and adults are nausea, loss of appetite, aching muscles, and headache. The characteristic rash follows this or oral sores, malaise, and a low grade fever that signal the presence of the disease. Oral manifestations of the disease (enanthem) not uncommonly may precede the external rash (exanthem). In children, the illness is not usually preceded by prodromal symptoms, and the first sign is the rash or the spots in the oral cavity. The rash begins as small red dots on the face, scalp, torso, upper arms and legs; progressing over 10-12 hours to small bumps, blisters and pustules; followed by umbilication and the formation of scabs. $[17,18,19]$.

At the blister stage, intense itching is usually present. Blisters may also occur on the palms, soles, and genital area. Commonly, visible evidence of the disease develops in the oral cavity and tonsil areas in the form of small ulcers, which can be painful or itchy, or both; this enanthem (internal rash) can precede the exanthem (external rash) by 1 to 3 days or can be concurrent. These symptoms of chickenpox appear 10 to 21 days after exposure to a contagious person. Adults may have a more widespread rash and longer fever, and they are more likely to experience complications, such as varicella pneumonia,[20,21]. Authors like $[17 ; 18 ; 19 ; 22 ; 23]$ had made more contribution in health regarding epidemiology and public health respectively.

Because watery nasal discharge containing live virus usually precedes both exanthem (external rash) and enanthem (oral ulcers) by 1 to 2 days, the infected person actually becomes contagious one to two days 
before recognition of the disease. Contagiousness persists until all vesicular lesions have become dry crusts (scabs), which usually entail four or five days, by which time nasal shedding of live virus ceases. The condition usually resolves by itself within a couple of weeks. The rash may, however, last for up to one month.

\section{METHODOLOGY}

Here are the method and the process of computing the values here in this chapter. Therefore, below is the data obtained from the primary health care management board Yobe State, an epidermis of Chickenpox in Nayinawa Ward, Damaturu Local Government Yobe State in the months of August 2018.

\section{MATHEMATICAL MODELING}

The mathematical description of system of phenomena is called a mathematical modeling and usually constructed with certain goals in mind for example the spread of Chickenpox disease in a certain community of host at a certain in rate in time can reliably assure that the rate at which the Chickenpox spread is directly proportional to both the infected and non-infected person. Written as:

$$
\frac{d n}{d t} \propto y(y-N)=\frac{d n}{d t}=k y(N-y)
$$

Where:

$\mathbf{N}=$ number of population

$\mathbf{k}=$ proportional factor

$$
\begin{aligned}
& \text { e.g. } 4 x^{2} y^{11}+y^{1} x+3 y=0 \ldots \\
& y(4)=y s-y(c) y^{1}(4)=-\frac{3}{64}
\end{aligned}
$$

$\mathbf{Y}=$ infected person and $\mathbf{N}-\mathbf{Y}=$ non-infected persons and $\mathbf{K}$ is always greater than 0 i.e. $\mathbf{K}>0$.

\section{DIFFERENTIAL EQUATION}

A differential equation is a mathematical equation that relates some function with it derivative in application the function usually represent physical quantities, the derivatives represent their rate of change and the differential equation define the relationship between them. Differential equation fist come into existence with the intervention of calculus by Newton and Leibniz.

\section{INITIAL CONDITIONS}

Initial conditions are condition or set of condition on the solution that will allow us to determine which solution that ne are after. Initial condition are of the form $\mathrm{y}\left(\mathrm{t}_{0}\right)=y_{0}$ and $y^{\mathrm{k}}\left(\mathrm{t}_{\mathrm{o}}\right)=y k$ in other word initial condition are values of the solution and it decretive at a specific points. Differential equations are urine hence only cone solution will meet the often conditions.

The member of initial conditions that required for a given differential equation mill depend upon the order of the differential equations.

\section{INITIAL VALUE PROBLEM}

An initial value problem is a differential equation along in the appropriate number of initial condition.

\section{VARIABLE SEPARABLE METHOD}

The differential equation $\frac{d n}{d t}=k y(N-y)$ is called first order differential equation and can be solved by separable method, as follows:

$$
\begin{gathered}
d n / d t=k y(N-y) \\
\frac{d N}{y(N-y)}=k d t \\
\therefore \int \frac{d N}{y(N-y)} \int k d t \\
\int \frac{1}{y(N-y)} d N=k d t+c \\
B u t \\
\frac{1}{y N-y)}=\frac{A}{y}+\frac{B}{N-y} \\
\frac{A(N-y)+B y}{y(N-y)}=\frac{1}{y(N-y)} \\
\therefore A(N-y)+B y=1
\end{gathered}
$$

Let

$$
\begin{array}{cc}
A(N-N)+B N=1 & y=N \\
A(0)+B N=1 & \\
B N=1, B=\frac{1}{N} &
\end{array}
$$


also,

$$
\begin{gathered}
A(N-N)+B N=1 \\
B N=1, B=\frac{1}{N}
\end{gathered}
$$

Also,

Let $\mathrm{y}=0$

Hence,

$$
\begin{gathered}
A(N-0)+B(0)=1 \\
A N+0=1 \\
A N=1, A=\frac{1}{N}
\end{gathered}
$$

$$
\frac{A}{y}+\frac{B}{N-y}=\frac{\frac{1}{N}}{y}+\frac{\frac{1}{N}}{N-y}
$$

Therefore,

$$
\begin{gathered}
\int \frac{1}{y(N-y} d N=\int\left(\frac{A}{y}+\frac{B}{N-y}\right) d N=\int\left(\frac{\frac{1}{N}}{y}+\frac{\frac{1}{N}}{N-y}\right) d N=\frac{1}{N} \int \frac{1}{y}+\frac{1}{N-y}=k t+c \\
\frac{1}{\mathrm{~N}}(1 \mathrm{n}|N| 1 \mathrm{n}|N-y|)=k t+c \\
1 \mathrm{n}\left|\frac{N}{N-y}\right|=N e^{k t}+c \\
\left.\frac{N}{N-y} N e^{k t}+c \ldots \ldots \ldots \ldots \ldots . . .12 .4\right)
\end{gathered}
$$

For some constant $\mathrm{N}$ and $\mathrm{K}$ substituting the value of initial conditions in (4) above lead us to logistic equation.

\section{LOGISTIC MODEL EQUATION}

$$
\frac{d p}{d t} \propto p ; \frac{d p}{d t}=k p \text { for some } k>0
$$

If we want the growth rate to approach zero as $\mathrm{p}$ approach the maximum carrying capacity we can introduce a limiting factor of M-P

$$
\therefore \frac{d p}{d t}=k p
$$

This is the logistic differential equation $\frac{d p}{d t}=p(a-b p)$ with a solution

$$
\begin{aligned}
& p(t)=\frac{a c e^{a t}}{1=b c e^{a t}}=\frac{a c e^{a t}}{e^{a t}\left(e^{-a t}+b c\right.}=\frac{a c}{b c+e^{-a t}} \\
& p(t)=\frac{a c}{b c+e^{-a t}} \ldots \ldots \ldots \ldots \ldots \ldots \text { (2.6) } \\
& \quad \text { if } t=0 \text { then } p(0)=p_{o} p_{0} \neq \frac{a}{b} \text { wefind }=\frac{p_{0}}{a-b p_{0}} \text { and so after substituting }
\end{aligned}
$$

And simplifying the solution becomes

$$
p(t)=\frac{a p_{0}}{b p_{0+\left(a-b p_{0}\right) e^{-a t}}} .
$$

where $\mathrm{A}$ and $\mathrm{B}$

Constant with $\mathrm{a}>\mathrm{b}$ at $\mathrm{t}=0$

$p(t)=\frac{a c}{b c+e^{-a t}} a t, t=0$

$\mathrm{P}(0)=p_{0}$ And

$$
\begin{aligned}
& p_{0}= \frac{a c}{b c_{+} e^{0}} p_{0}=\frac{a c}{b c+1} \\
& p_{0}(b c+1)=a c
\end{aligned}
$$


Substituting the value of $\mathrm{C}$ into equation $(2,6)$

$$
\begin{gathered}
p_{0} b c+p_{0}=a c \\
p_{0} b c+a c=-p_{0} \\
a c-p_{0} b c=p_{0} \\
c=\frac{p_{0}}{a-p_{0} b} \\
c\left(a-p_{0} b\right)=p_{0}
\end{gathered}
$$

$$
\begin{aligned}
& p_{t}=\frac{a c}{b c+e^{-a t}}=a\left(\frac{p_{0}}{a-b p_{0}}\right) \div b\left(\frac{p_{0}}{a-p_{0} b}\right)+\frac{e^{-a t}}{1} \\
& \frac{a p_{0}}{a-b p_{0}} \div \frac{b p_{0}+\left(a-p_{0} b\right) e^{-a t}}{a-p_{0} b} \\
& p_{(t)}=\frac{a p_{0}}{a-b p_{0}} \times \frac{a-b p_{0}}{b p_{0}+\left(a-p_{0} b\right) e^{-a t}}
\end{aligned}
$$

Multiply equation $(2,7)$ by $\frac{e^{a t}}{e^{a t}}=1$

$$
p_{t}=\frac{a p_{0} e^{a t}}{b p_{0} e^{a t}+\left(a-p_{0} b\right)} \text {. }
$$

Where,

$\boldsymbol{p}_{\mathbf{0}}=$ Population Of the host community at the initial time

$\boldsymbol{B}=$ the final population after some long time also called the maximum carrying capacity with limit growth

$\boldsymbol{A}=$ the initial growth rate

$\boldsymbol{t}=$ time measure on days

\section{DETERMINSTIC MODELLING TECHNICS}

The first steps of deterministic modeling consist of having a complete and realistic picture of the biology of the diseases. Example, duration of the period of infection, incubation periods status after infection.
The second step is to collect the data on the demographic epidemiology and the characteristic of the infection (transmission rate) and the population (birth rate and death rate).

The third step a parsimonious model is selected

\section{RESULT}

In this chapter we are to use the model formulated in chapter three to compute the result at various time and the result obtained can be discussed to enable us depict the epidemic or otherwise.

Table-1: Show the Susceptible, infected people and the time period

\begin{tabular}{|l|l|l|l|l|l|l|}
\hline T & 0 & 1 & 2 & 3 & 4 & 5 \\
\hline S & 12,000 & 11,991 & 11,920 & 11,318 & 7,790 & 2,051 \\
\hline I & 1 & 9 & 80 & 682 & 4,210 & 9,949 \\
\hline
\end{tabular}

Where,

$\mathbf{S}=$ Susceptible people

$\mathbf{t}=$ time period

$\mathbf{I}=$ infected person

Since the rate of speed of Chickenpox contagious disease is directly proportional to the infected and no-infected person, the village (Nayinawa) has a total population of twelve thousand $(12,000)$ people. If the infected people is represented as $\mathrm{N}$ then the Non-infected people is $12000-\mathrm{N}$ "

Hence $\frac{d N}{d t} \alpha N(2,000-N)$

$$
\frac{d N}{d t}=k N(12,000-N)
$$

Variable Separable

$$
\frac{d N}{N(12000-N)} k d t
$$

Integrating both sides

$$
\int \frac{d N}{N(12000-N)}=k \int d t
$$




$$
\begin{aligned}
& \therefore \frac{1}{N(12000-N)}=\frac{A}{N}+\frac{B}{12000-N} \\
& \frac{A}{N}+\frac{B}{12000-N}=\frac{1}{N(12000-N} \\
& \frac{A(12000-N)+B N}{N(12000-N)}=\frac{1 B}{N(12000-N)}
\end{aligned}
$$

$\mathrm{A}(12000-\mathrm{N})+\mathrm{BN}=1$

let $\mathrm{N}=12,000$

A $(12,000+2000)+12000 \mathrm{~B}=1$

$0+12000 \mathrm{~B}=1$

$$
B=\frac{1}{12000}
$$

Again let $\mathrm{N}=0$

$$
\begin{aligned}
& A(12000-N)+B N=1 \\
& A(12000-)+B(0)=1,12000 A+0=1 \\
& 12000 A=1, A=\frac{1}{12000} \\
& \therefore \frac{A}{N}+\frac{B}{12000-N}=\frac{1}{\frac{12000}{(N)}}+\frac{1}{\frac{12000}{12000-N}} \\
& \left.\int \frac{1}{\frac{12000}{N}} d N+\int \frac{1}{\frac{12000}{12000-N}} d N\right)=K \int d t \\
& \frac{1}{12000}\left[\int \frac{1}{N} d N+\int \frac{1}{12000-N} d N\right]=k t+c \\
& \frac{1}{12000}(1 \mathrm{n} / N /-h / 12000-N /)+D=k t+c \\
& \frac{1}{12000}(1 \mathrm{n} / N /-1 \mathrm{n} / 12000-N /)+D=k t+c \\
& 1 \mathrm{n} / N /-1 \mathrm{n} / 12000-N /=(k t+c) 12000 \\
& \left|\frac{N}{12000-N}\right|=R_{1} t+c_{1}
\end{aligned}
$$

Where $\mathbf{k}$ is constant and $\mathbf{N}$ is always between 1 and 12000 we can drop the absolute value.

$$
\text { 1n }\left|\frac{N}{12000-N}\right|=R_{1} t+c_{1}
$$

At the initial condition $\mathrm{N}(\mathrm{t})=\mathrm{N} \mathrm{N}(0)=1$

$$
\ln \left|\frac{N}{12000-N}\right|
$$

$$
\text { 1n }\left|\frac{N}{12000-N}\right|=R_{1} t+c_{1}
$$

Again N (2) $=80$ implies that;

$$
\ln \left|\frac{80}{12000-80}\right|=2 k++\left|\frac{1}{11,999}\right|
$$

From here we can plug in the values of $t$ and solve for the values of $\mathrm{N}$, by substituting these initial condition it lead is to logistic equation (3.0) chapter two. This means that it will first cause on exponential increase, which will slow down and eventually level it.

$\mathrm{N}(2)=80$ from the Table- 1

The logistic differential equation model was originally put forward by Verhust in 1845 which is an ordinary differential equation used to describe the selfgrowth characteristics of populations. The model differential equation is as follows:
$P(t)=\frac{k p_{0} e^{n}}{k+p_{0}\left(e^{n_{-}}\right.}$Logistic equation

$$
\begin{gathered}
\therefore 80=\frac{12000 \times 1 \times e^{r t}}{12000+1\left(e^{2 r}-1\right)} \\
80=\frac{12000 \times^{2 r}}{12000+1 e^{2 r}-1} \Rightarrow 80=\frac{12000 e^{2 r}}{11,999+e^{2 r}} \\
8 \mathrm{~T}\left(11,999+e^{2 r}\right)=12000 \mathrm{e}^{2 r} \\
959920+80 \mathrm{e}^{2 r}=12000 \mathrm{e}^{2 r} \\
959920=(12000-0) \mathrm{e}^{2 r}
\end{gathered}
$$




$$
\begin{array}{r}
\frac{959920}{11920} \mathrm{e}^{2 r} \\
80.53=\mathrm{e}^{2 r} \\
\ln 80.53=2 r \\
r \frac{1}{2} \ln 80.53 \\
\therefore p(t)=\frac{k p_{0} \mathrm{e}^{\frac{1}{2} \ln 80.53 t}}{k p_{0}\left(\mathrm{e}^{\frac{1}{2} \ln 80.53 t}\right)} \ldots \ldots \ldots . .
\end{array}
$$

The above equation is the general model depicting the number of infected popular in that host community at any point in time,

In the Table-1 the letter's' means the susceptible people (meaning the people that can be infected in the community, the rate at which (s) changes with time in the host community, the rate at which (s) changes with time in the host community once the epidemic erupted is $S$ and it is always negative, expressed as $\mathrm{S}=$ total population + the rate of change in susceptible $\mathrm{x}$ the times (in days).

Also 'I' means the infected people, the change in the number of infected people in the community of host with is ' $\mathrm{I}$ '.

Since,

$$
p(t)=\frac{k p_{0} \mathrm{e}^{\frac{1}{2} \ln 80.53(t)}}{k+p_{0}\left(\mathrm{e}^{\frac{1}{2} \ln 80.53(t)}\right)}
$$

The parameters of the logistic model fitting results of varicella epidemic in summer-autumn and winter-spring between 2005 and 2013 in Damaturu.

\begin{tabular}{|l|l|l|l|}
\hline Time & Parameter $\boldsymbol{k}$ & Parameter $\boldsymbol{N}$ & Parameter $\boldsymbol{c}$ \\
\hline Summer-autumn & & & \\
\hline 2010 & 0.346 & 2162 & -4.68 \\
\hline 2011 & 0.317 & 2002 & -4.07 \\
\hline 2012 & 0.309 & 2415 & -4.47 \\
\hline 2013 & 0.304 & 3235 & -4.16 \\
\hline 2014 & 0.311 & 2776 & -4.18 \\
\hline 2015 & 0.271 & 2518 & -3.83 \\
\hline 2016 & 0.262 & 2510 & -4.06 \\
\hline 2017 & 0.290 & 2697 & -3.84 \\
\hline 2018 & 0.318 & 2399 & -4.05 \\
\hline 2019 & 0.336 & 4880 & -4.37 \\
\hline Winter-spring & & & \\
\hline 2010 & 0.341 & 1642 & -4.08 \\
\hline 2011 & 0.299 & 1291 & -3.82 \\
\hline 2012 & 0.423 & 2522 & -4.55 \\
\hline 2013 & 0.338 & 2858 & -3.92 \\
\hline 2014 & 0.351 & 3179 & -4.34 \\
\hline 2015 & 0.362 & 2804 & -4.13 \\
\hline 2016 & 0.370 & 4348 & -4.98 \\
\hline 2017 & 0.384 & 3425 & -4.85 \\
\hline 2018 & 0.415 & 3934 & -4.49 \\
\hline 2019 & 0.356 & 3679 & -3.94 \\
\hline & & & \\
\hline
\end{tabular}

From equation $(3,3)$ the result can be computed as follows;

When the time $(\mathrm{t}=0)$ is zero

$$
\begin{gathered}
\mathrm{p}(\mathrm{t})=p(t)=\frac{k p_{0} \mathrm{e}^{1 / 2 \ln 80.53(t)}}{k+p_{0}\left(\mathrm{e}^{1 / 2 \ln 80.53(t)}\right)} \\
\mathrm{p}(0)=\frac{12000 \times 1 \times e^{1 / 21 \mathrm{n} 80.53(2)}}{12000+e^{1 / 21 \mathrm{n} 80.53(2)}}=\frac{12000 e^{4.3886}}{12000-1+e^{4.3886}}=\frac{12000 \times 1}{12000+1-1}=1(\text { person infected })
\end{gathered}
$$

Initially,

When the time $(\mathrm{t})$ is one $(\mathrm{t}=1)$.

$$
\begin{aligned}
& p(t)=\frac{12000 \times 1 \times e^{1 / 21 \mathrm{n} 80.53(2)}}{12000+e^{1 / 21 \mathrm{n} 80.53(2)}}=\frac{12000 e^{4.3886}}{12000-1+e^{4.3886}} \\
& \frac{12000 \times 80.5276}{11,999+80.5276}=\frac{966,331.2}{12,079.5276} 79.997 \cong 79.999 \text { people were infected the second day. }
\end{aligned}
$$

When $\mathrm{t}=3$ 


$$
\begin{aligned}
& \mathrm{p}(3)=\frac{12000 \times 1 e^{1 / 21 \mathrm{n} 80.53(3)}}{12000+1\left(e^{1 / 21 \mathrm{n} 80.53(3)}\right)}=\frac{12000 \times e^{6.5829}}{11,999+e^{6.5829}}=\frac{12000 \times 722.6319}{11,999+722.6319}=\frac{8,671,582.8}{12,791.6319} \\
& =681.6408 \cong 682 \text { people were infected }
\end{aligned}
$$

When $\mathrm{t}=4$

$$
\mathrm{P}(4)=\frac{12000 \times 1 \times e^{1 / 21 \mathrm{n} 80.53(4)}}{11,9999+6,484.6945}=\frac{77,816,335.02}{18,483.69458}=4,209.998 \cong 4,210 \text { people were infected }
$$

Below is the table showing the computed values of the infected and non-infected person in the community of host over a short period of time by the model.

Table-1:

\begin{tabular}{|l|l|l|l|l|c|}
\hline $\mathbf{T}$ & \multicolumn{1}{|c|}{$\mathbf{0}$} & \multicolumn{1}{|c|}{$\mathbf{1}$} & \multicolumn{1}{|c|}{$\mathbf{2}$} & \multicolumn{1}{|c|}{$\mathbf{3}$} & $\mathbf{4}$ \\
\hline $\mathrm{S}$ & 12,000 & 11,991 & 11,950 & 11,318 & 7,790 \\
\hline $\mathrm{I}$ & 1 & 9 & 80 & 682 & 4,210 \\
\hline
\end{tabular}

Based on the above table, the value of infected people van be plotted against time period and the values of noninfected can also be plotted against the period.

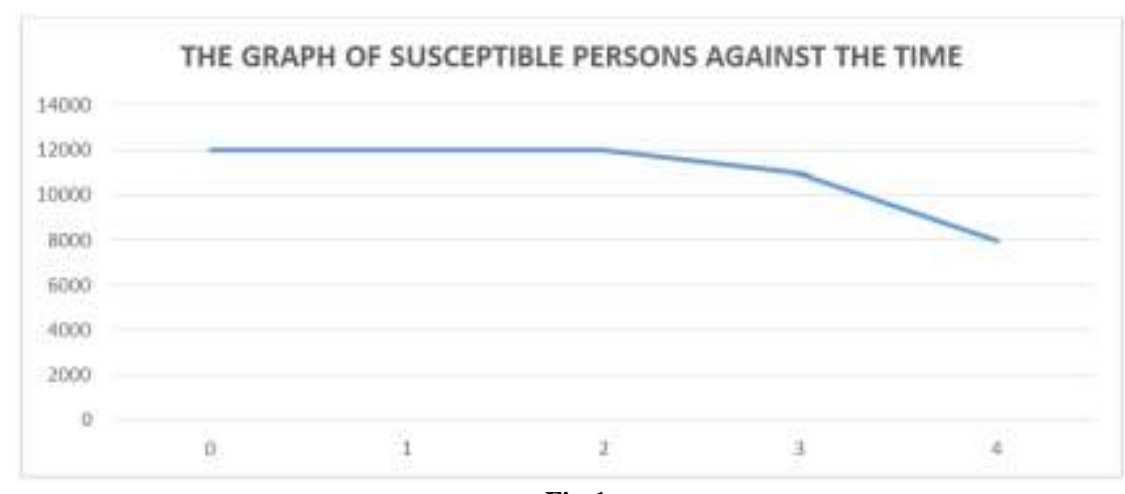

Fig-1

However, Table-1 and the above graph depicts that epidemics has set up and level of the epidemic is totally at the final stage affecting on average of 996. People in just five days, that is it infected positive steady from the onset and later it exponentially increase causing a very large damage to the community. In addition, the graph below depicted the non-infected people against the time in the same community of host at that period in time.

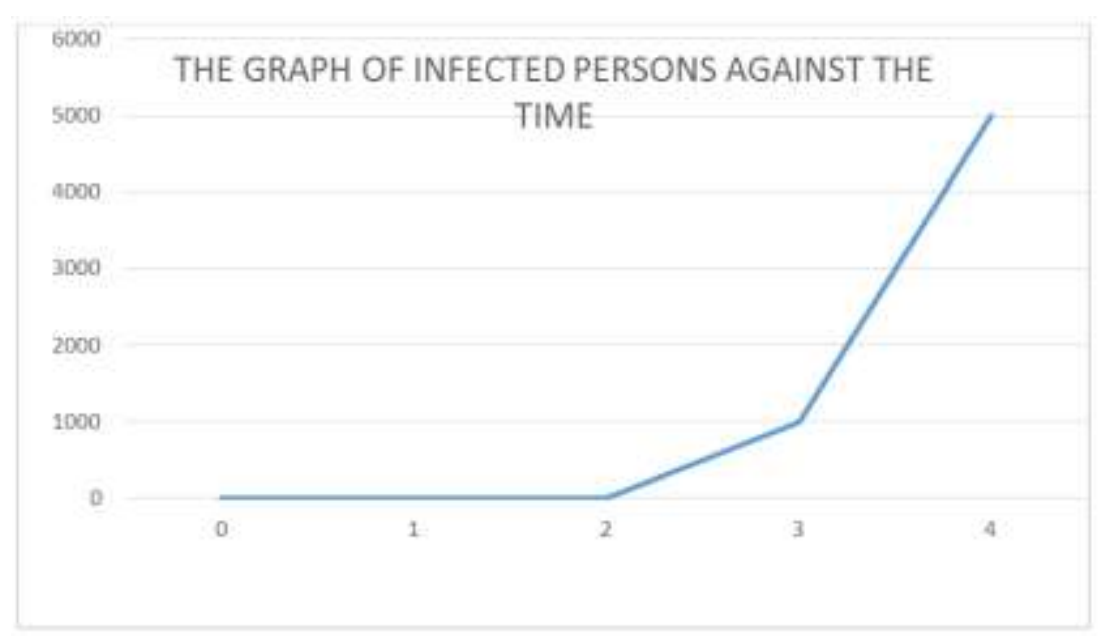

Fig-2

According to the graph sketched above, there is a decline in the number of non-infected person in that 
community of host, although the number of noninfected (12000-N) decline steady and later reduces sharply showing a threat to the community.

\section{DISCUSSION}

Chickenpox is distributed globally, which is a common infectious disease among children. In addition, it has a very strong ability to transmit among the population, and can affect nearly $90 \%$ of children. Nigeria has begun to report varicella outbreaks through the 'Nigeria Disease Prevention and Control Information System' since 2005.

Although varicella-infected patients are more common in light and moderate cases, if left untreated, the infection can lead to post-herpetic neuralgia, bacterial infections and, ultimately, death. At present, there is relatively less research on varicella, and the measures of prevention still stay in the stage of control and symptomatic treatment.

Many studies showed that there are many models for the prevention and prediction of infectious diseases, such as time-series models, grey models and SIS models based on complex network theory. At the same time, studies have shown that the logistic differential equation model can also be used to fit and predict the epidemic situation of infectious disease, which can also predict the early warning period for seasonal infectious diseases.

In this work, the epidemiological characteristics of varicella were studied by logistic differential equation model and the early warning week was obtained. The results show that chickenpox in Damaturu City has obvious periodicity and seasonality, and the epidemic cycle starts from the 8th week of each year to the 7 th week of the next year. Therefore, it is recommended that chickenpox surveillance and statistical methods should be adapted for their prevalence characteristics. In addition, the research results show that the logistic model can well fit the epidemic pattern of chickenpox, and we can get the 'acceleration week' of winter-spring and summerautumn, respectively, each year according to the model. However, it can be seen that the epidemic has reached a high level when the epidemic has accelerated, which means that there will be a lag if the warning measures were taken during the acceleration week. Therefore, 'recommended warning week', calculated based on the standard deviation, is a better-recommended warning time.

In practice, it usually takes a certain amount of time to show the effect of the implementation of interventions. Therefore, the varicella intervention usually needs to be planned and implemented early. This means that the early warning signal of chickenpox needs to be issued in advance to provide sufficient preparation time for the health decision-making and the measures implementation departments. The advanced warning week' proposed in this, study is 2 weeks prior to the epidemic season, which is the timing that the rate of change of the epidemic progresses from slow to fast. This can provide sufficient time for preparation and implementation of interventions.

In this study, the logistic differential equation model was used to analyze the prevalence and periodicity of varicella epidemics in Damaturu town from the eight week of 2008 to the 2019; to establish the logistic differential equation model in each cycle according to the epidemic pattern of chickenpox, and further calculation of the epidemic development rate parameter $k$ and the theoretical value $N$ of cumulative cases at the end of the epidemic cycle through the actual weekly epidemiological data. In addition, we get the 'epidemic acceleration week' and 'recommended warning week' of chickenpox, which provided a scientific basis for better understanding of the epidemic characteristics and early warning work of chickenpox. Of note, although our study has achieved its original purpose, it still has certain limitations due to the limitations of the model and the data availability. First, the model is based on epidemiological data analysis without considering the characteristics and the climatic condition of the disease, omitting them from the model may have slightly affected the simulated data. Second, the effect of the implementation of the interventions were not added when the warning week determined. In the future, these dynamic characteristics, intervention effects and climatic factors can be added to conduct indepth studies to provide more detailed evidence for the prevention and control of chickenpox.

\section{CONCLUSION}

Based on the findings so far, an epidemic has set up affecting the positive fraction of the community of host with an average of 996 infected person daily. The epidemic level was slow in the first place but later it increases exponentially indicating a sign of danger to the said community of host.

The varicella in Damaturu has a clear cycle and seasonality, and there are two peaks each year: winter-spring and summer-autumn. What is more, the logistic model can be well fitted with varicella data. However, the early warning week obtained by the model shows that the warning has lagged. Therefore, adjusting by the standard deviation can give a betterrecommended warning time, and this has a good effect on preventing chickenpox outbreaks and can provide a scientific evidence for the clinical and early warning work of Varicella.

\section{RECOMMENDATION}

Sequel to the finding obtained in this study it is obvious that the results obtained have depicted the number of people likely to be infected over a period of time and make a reasonable for best of how many 
people to be infected in a certain time to enable make a proper decision and supply in case of an outbreak (epidemics) like this in a giver community of host with known population number.

Although the study has not make it to our consumption the death that occurred since inception of the epidemic the number of recovered people and the inclining a declining, in the trend of their immune system during the epidemic when on treatment. Further study could be conducted by any interested candidate to make clarification these not - known parameter enlisted above by the study.

A study of this nature need data from the host community which were normally monitored and checked by health agencies a difficulties is often encountered while trying to collect the data for the project. In Conclusion, we would like to say that since there is an outbreak in the said community of host a special care unit be built by the government in at least every village unit of the local government so that we can get proper health care.

\section{REFERENCE}

1. Lämmer, S., \& Helbing, D. (2008). Self-control of traffic lights and vehicle flows in urban road networks. Journal of Statistical Mechanics: Theory and Experiment, $2008(04), \mathrm{P} 04019$.

2. Wilson, S. (2008). Research is ceremony: Indigenous research methods.

3. Kapoor, H., Akerman, M., Justus, S., Ferguson, J. C., Korsunsky, Y., Gallo, P., ... \& Xu, W. (2007). U.S. Patent Application No. 11/610,296.

4. Hatchett, R. J., Mecher, C. E., \& Lipsitch, M. (2007). Public health interventions and epidemic intensity during the 1918 influenza pandemic. Proceedings of the National Academy of Sciences, 104(18), 7582-7587.

5. Briët, O. J., Amerasinghe, P. H., \& Vounatsou, P. (2013). Generalized seasonal autoregressive integrated moving average models for count data with application to malaria time series with low case numbers. PloS one, 8(6):e65761.

6. Wang, Y. W., Shen, Z. Z., \& Jiang, Y. (2018). Comparison of ARIMA and GM $(1,1)$ models for prediction of hepatitis B in China. PloS one, 13(9): 0201987.

7. Hammoudi, D., Moubareck, C. A., Aires, J., Adaime, A., Barakat, A., Fayad, N., ... \& Suleiman, M. (2014). Countrywide spread of OXA-48 carbapenemase in Lebanon: surveillance and genetic characterization of carbapenem-nonsusceptible Enterobacteriaceae in 10 hospitals over a one-year period. International Journal of Infectious Diseases, 29, 139-144.

8. Ogunjimi, B., Willem, L., Beutels, P., \& Hens, N. (2015). Integrating between-host transmission and within-host immunity to analyze the impact of varicella vaccination on zoster. Elife, 4, e07116.
9. Bekoe, C. (2015). The SIR Model and the 2014 Ebola Virus Disease Outbreak in Guinea, Liberia and Sierra Leone. International Journal of Applied, 6(2), 11.

10. Inyama, C. N., Mbagwu, F. N., \& Duru, C. M. (2016). Taxonomic Relationship on Some Chrysophyllum Species based on Anatomical Studies. Med Aromat Plants, 5(227), 2167-0412.

11. Engel, R. J., \& Schutt, R. K. (2016). The practice of research in social work. Sage Publications.

12. Tracy, P. (2016). What is a smart building and how can it benefit you.

13. Ogunjimi, B., Van den Bergh, J., Meysman, P., Heynderickx, S., Bergs, K., Jansens, H., ... \& Cools, N. (2017). Multidisciplinary study of the secondary immune response in grandparents reexposed to chickenpox. Scientific reports, 7(1), 111.

14. Chapman, C. (2017). Eighteenth-century Women Artists: Their Trials, Tribulations \& Triumphs. Unicorn.

15. Jonathan Cohen, Consultant in General Paediatrics and Paediatric Infectious Diseases ${ }^{\#}$ and Judith Breuer, Professor of Virology 2019.

16. Rafferty, E., McDonald, W., Qian, W., Osgood, N. D., \& Doroshenko, A. (2018). Evaluation of the effect of chickenpox vaccination on shingles epidemiology using agent-based modeling. PeerJ, 6, e5012.

17. Umar Yusuf Madaki, MAB and AbdulRahman Malik (2018), Statistical Analysis on Diabetic Patients Among Adults in Damaturu, Nigeria. International Journal of Innovative Research and Development, 7(10), pages 9.

18. Umar Yusuf Madaki, MAB and AbdulRahman Malik (2018), Prevalence of Bronchopulmonary Dyplasia Among Infants Using Logistic Regression Model for UMTH and UDUTH Hospitals in Nigeria. International Journal of Innovative Research and Development, 7(10), pages 12.

19. V.N. Maurya, M.U.Yusuf, V.V. Singh \& Babagana Modu (2015), Application of Discriminant Analysis on Bronchopulmonary Dyplasia Among Infants: A Case Study of UMTH and UDUTH Hospitals in Nigeria. American Journal of Theoretical and Applied Statistics, 4(2-1), pages 44-51.

20. V.V. Singh, M.U.Yusuf, \& Musa Chiwa Dalah (2019), Assessment of Diabetic Patients Among Adults in Maiduguri, Using Multivariate Discriminant Model. Juniters Publishers, 555754(1), pages 8 .

21. MRBA Bakar and M.U.Yusuf, (2016), Cure Models based on Weibull distribution with or without Covariates using Right Censored Data. Indian Journal of Science and Technology, 9(28), pages 12 .

22. MRBA Bakar and U.Y. Madaki, (2016), A Bayesian Estimation on Right Censored Survival 
Data With Mixture and Non-mixture Cure Fraction Models based on Beta-Weibull distribution. AIP Proceedings of the $2^{\text {nd }}$ International Conference on Mathematical Sciences and Statistics (ICMSS2016), 1739(1), pages 020079.
23. Madaki Umar Yusuf and MRBA Bakar, (2016), Survival Data with Incomplete Categorical Covariates, World Academy of Science Engineering and Technology, 2(6A), pages 6-12. 